\title{
Mixing study of a jet in crossflow using accurate thermal anemometry techniques
}

\author{
Jean-Paul MORO ${ }^{1}$, Pierre FOUGAIROLLE ${ }^{1}$, and Yves GAGNE ${ }^{2}$ \\ 1 CEA Grenoble 17 rue des Martyrs - 38054 Grenoble Cedex 9, France \\ Jean-Paul.Moro@cea.fr \& Pierre.Fougairolles@cea.fr \\ 2 Université Joseph Fourier - BP 53 - 38041 Grenoble Cedex 9, France \\ Yves. Gagne@hmg. inpg. fr
}

The objective of this experimental work is to focus on the turbulent mixing between a pipeflow exiting heated jet and a confined crossflow, with both kinematic and passive scalar measurements in order to characterize its statistical properties (energy spectra, pdf, skewness, anisotropy...).

The experimental set-up consists of a grid-turbulence flow in a rectangular wind tunnel $\left(60 \times 50 \mathrm{~cm}^{2}\right.$ in section and $5 \mathrm{~m}$ long) in which emerges a perpendicular rectangular pipeflow $\left(8 \times 5 \mathrm{~cm}^{2}\right.$ section, equivalent to an hydraulic diameter $D_{h}=6.2 \mathrm{~cm}$ for a circular jet). Ranges of velocity are $U=0-20 \mathrm{~m} \cdot \mathrm{s}^{-1}$ and $U_{j}=0-40 \mathrm{~m} \cdot \mathrm{s}^{-1}$ for the crossflow and the jet respectively. Measurements are performed with classical hot-wire anemometry and cold-wire thermometry but with a peculiar care in the apparatus construction. A unique and reproductible technique allows us to make probes with Wollaston wire of $500 \mathrm{~nm}$ diameter, which can be used to measure velocity fluctuations as well as temperature ones. To avoid bad tuning effects, we have chosen a constant voltage anemometer home-made in CEA (SSTH/LIEX) on the basis of the method developed by Sarma [1] and Comte-Bellot \& al. Our thermo-anemometers lead to a signal to noise ratio up to 1000 for the velocity and a sensitivity of $0.015^{\circ} \mathrm{C}$ for the temperature.

Temperature maps (in the scalar passive case, $\Delta T=T_{j}-T=7.5^{\circ} \mathrm{C}$ ) with a momentum ratio $r=U_{j} / U$ of 10 , measured $3 D_{h}$ downstream of the jet inlet, are displayed on figure 1. It reveals the global signature of the counterrotating vortex pair (CVP) analogously to the round jet case (cf. Smith and Mungal [2] and Fric [3]).

Figure 2 shows mean temperature profiles measured in the crossflow channel at several sections downstream of the jet inlet. We clearly observe that there is no self-similarity between these latters. That can be explained by the jet interaction with the opposite boundary wall since $8 D_{h}$. Even for distances under $5 D_{h}$, the shapes of the curves seem different. 
(a)

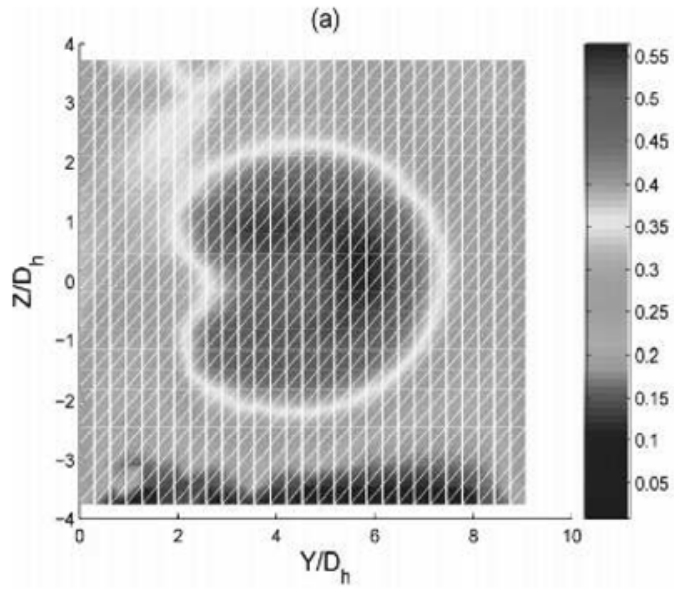

(b)

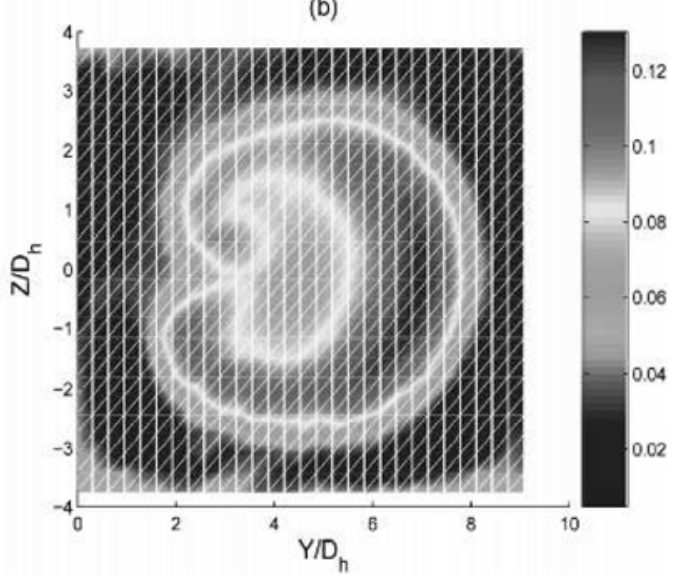

Fig. 1. Temperature field (normalized by $\Delta T$ ) interpolated on 29x24 measurement points in the section located at $3 D_{h}$ downstream of the jet inlet. a) Average value b) $\mathrm{rms}$

Figure 3 shows the temperature pdf locally obtained on the center of the jet hot spot in the same sections. Despite the lack of similarity at large scales, the statistics exhibit some common features ; particularly, they are skewed whatever the distance from the jet inlet.

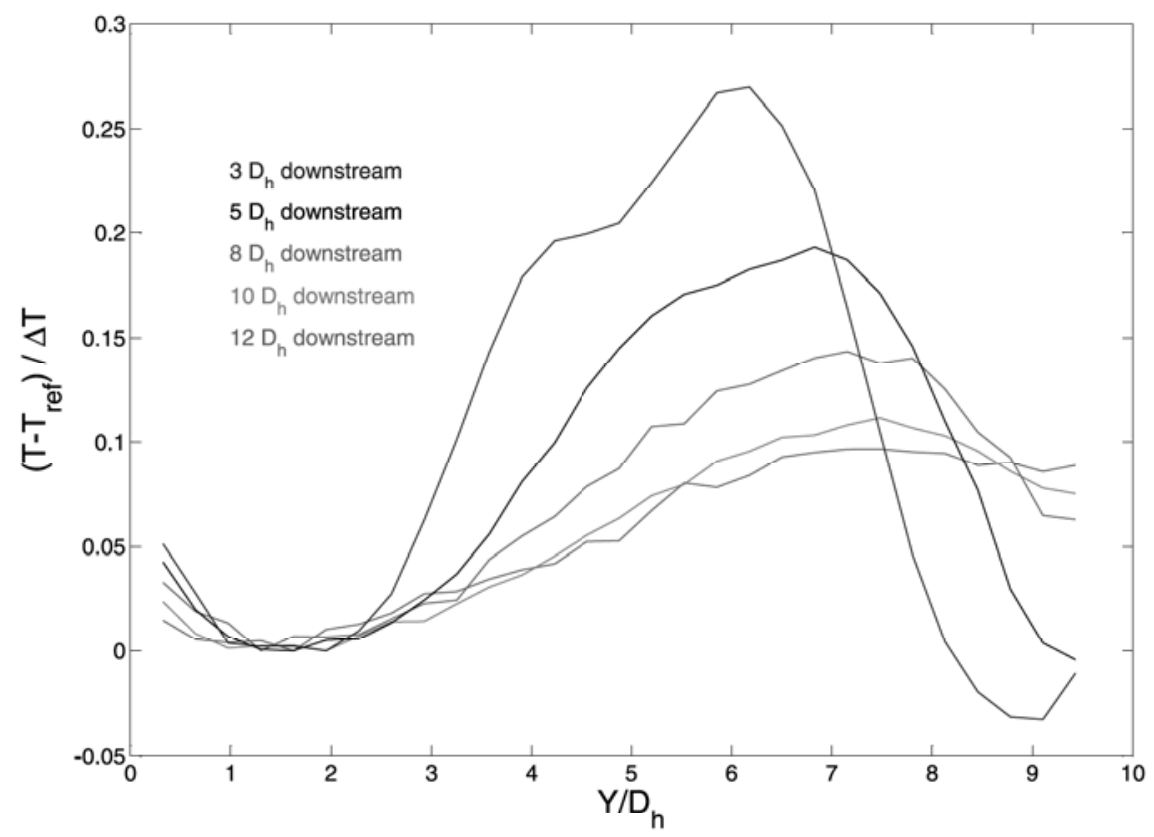

Fig. 2. Normalized mean temperature profiles measured on the center of the jet inlet $\left(Z / D_{h}=0\right)$ at several sections downstream. Jet emerges from $Y / D_{h}=0$ and the opposite wall is situated at $Y / D_{h}=9.7$. 
The merging of the curves for hot fluctuations suggests a good mixing of these ones. On the other side, the quasi-exponential negative wings reveals the existence of "cold" fluid structures coming from the crossflow, which are not yet uniformly mixed. These wing become longer as the cold fluctutations diffuse. Such statistics correspond to the typical "ramp", characteristic of a temperature mixing signal.

Further investigations will explore statistical properties of the external side of this transversal hot jet, especially its interaction with the crossflow channel wall, and of its internal side which is mainly affected by the wake of the jet inlet.

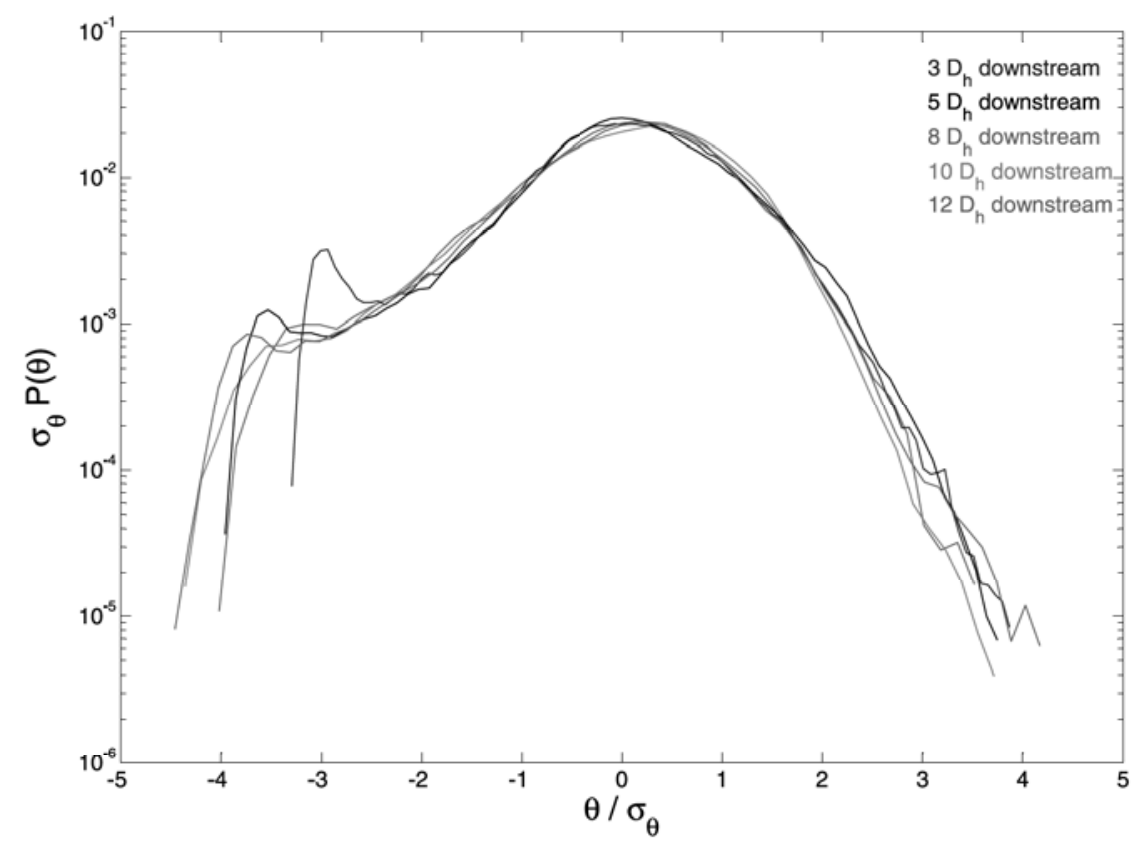

Fig. 3. Pdf of the temperature fluctuations $\theta$ normalized by its standard deviation, measured in the center of the jet hot spot at several sections downstream of the jet inlet.

\section{References}

1. G.R. Sarma : Review of scientific instruments Vol. $69 \mathrm{~N}^{\circ} 6$ (1998)

2. S.H. Smith, M.G. Mungal : J. Fluid Mech. 357, 83-122 (1998)

3. T.F. Fric : Structure in the near field of the transverse jet. PhD Thesis, California Institute of Technology (1990) 